\title{
Self-harm in children and adolescents by ethnic group: an observational cohort study from the Multicentre Study of Self-Harm in England
} DOI:

10.1016/S2352-4642(21)00239-X

\section{Document Version}

Accepted author manuscript

Link to publication record in Manchester Research Explorer

Citation for published version (APA):

Farooq, B., Clements, C., Hawton, K., Geulayov, G., Casey, D., Patel, A., Kelly, S., Townsend, E., Appleby, L., \& Kapur, N. (2021). Self-harm in children and adolescents by ethnic group: an observational cohort study from the Multicentre Study of Self-Harm in England. The Lancet Child \& Adolescent Health. https://doi.org/10.1016/S23524642(21)00239-X

\section{Published in:}

The Lancet Child \& Adolescent Health

\section{Citing this paper}

Please note that where the full-text provided on Manchester Research Explorer is the Author Accepted Manuscript or Proof version this may differ from the final Published version. If citing, it is advised that you check and use the publisher's definitive version.

\section{General rights}

Copyright and moral rights for the publications made accessible in the Research Explorer are retained by the authors and/or other copyright owners and it is a condition of accessing publications that users recognise and abide by the legal requirements associated with these rights.

\section{Takedown policy}

If you believe that this document breaches copyright please refer to the University of Manchester's Takedown Procedures [http://man.ac.uk/04Y6Bo] or contact uml.scholarlycommunications@manchester.ac.uk providing relevant details, so we can investigate your claim.

\section{OPEN ACCESS}




\section{Self-harm in children and adolescents by ethnic group: an observational cohort study from the Multicentre Study of Self-Harm in England}

Bushra Farooq, Caroline Clements, Keith Hawton, Galit Geulayov, Deborah Casey, Keith Waters, Jennifer Ness, Anita Patel, Samantha Kelly,

Ellen Townsend, Louis Appleby, Nav Kapur

\section{Summary}

Background Studies report an increasing incidence of self-harm in children and adolescents, but the extent to which this is seen in different ethnic groups is unclear. We aimed to investigate rates of emergency department presentations for self-harm in children and adolescents by ethnicity, as well as to examine their demographic characteristics, clinical characteristics, and outcomes.

Methods In this observational cohort study, we used data on hospital emergency department presentations for selfharm in children and adolescents aged 10-19 years between 2000 and 2016 from the Multicentre Study of Self-harm in England. This study collects data from five general hospitals in Manchester, Oxford, and Derby in the UK, and defines self-harm as any act of intentional self-injury or self-poisoning, regardless of intent. All children and adolescents aged 10-19 years for whom ethnicity data were available were included. Mortality follow-up was available through linkage with mortality records from the Office for National Statistics. Rates of self-harm over time, demographic and clinical characteristics, and self-harm methods were investigated by ethnic group. Risk of repeat self-harm and mortality following an initial presentation for self-harm was compared by ethnic group using Kaplan-Meier curves and Cox proportional hazards models.

Findings Of 14894 individuals who presented at hospitals with self-harm, 11906 had data for ethnicity, of whom $10211(85 \cdot 8 \%)$ were White, $344(2 \cdot 9 \%)$ were Black, 619 (5.2\%) were South Asian, and $732(6 \cdot 1 \%)$ were other non-White. Rates of self-harm were highest in White children and adolescents but increased between 2009 and 2016 in all ethnicities. Mean annual rates of self-harm per 100000 population were 574 for White, 225 for Black, 260 for South Asian, and 344 for other non-White groups. Increases in rates of self-harm between 2009 and 2016 appeared slightly greater in Black groups (incidence rate ratio 1. 07 [95\% CI 1.03-1·11]), South Asian groups (1.05 [1.01-1.09]), and other non-White groups $(1 \cdot 11[1 \cdot 06-1 \cdot 16])$ than in White groups $(1 \cdot 02[1 \cdot 00-1 \cdot 03])$. Children and adolescents from a minority ethnic background were more likely to live in areas of high deprivation and were less likely to receive a specialist psychosocial assessment than were White children and adolescents. Children and adolescents from minority ethnic groups were also less likely to repeat self-harm. However, there were no differences in suicide mortality by ethnic group, although the numbers were small.

Interpretation Minority ethnic children and adolescents accounted for an increased proportion of self-harm presentations to hospital over time compared with White ethnic groups. The minority ethnic groups also tended to be more socioeconomically disadvantaged and were less likely to receive a psychosocial assessment. Socioeconomic disparities need to be addressed, and equitable access to culturally sensitive comprehensive psychosocial assessments must be ensured.

Funding UK Department of Health and Social Care.

Copyright (C) 2021 Published by Elsevier Ltd. All rights reserved.

\section{Introduction}

Self-harm in children and adolescents is a major health and societal issue. ${ }^{1}$ Rates of self-harm during adolescence are increasing and estimates suggest that each year in England 200000 adolescents aged 12-17 years self-harm and do not come to the attention of clinical services, and that a further 21000 adolescents present to hospital following self-harm. ${ }^{1-3}$

Self-harm is a key area for action in the National Suicide Prevention Strategy for England. ${ }^{4}$ All-cause mortality following hospital presentation for self-harm in people aged $10-18$ years is $1.4 \%$, and 12 -month incidence of suicide in people who self-harm is 30 -times higher than in the general population in England. ${ }^{5}$ About $27 \%$ of people aged 10-18 years who present to hospital for self-harm present again at some point, and $17.7 \%$ of people aged 10-19 years repeat self-harm within 12-months of presenting to hospital. ${ }^{6,7}$

Self-harm is an indicator of distress with functions beyond those linked to suicidal intent. It is used variously
Lancet Child Adolesc Health 2021 Published Online September 20, 2021 https://doi.org/10.1016/ S2352-4642(21)00239-X See Online/Comment https://doi.org/10.1016/ S2352-4642(21)00303-5 Centre for Mental Health and Safety, School of Health Sciences, Manchester Academic Health Science Centre, The University of Manchester, Manchester, UK (B Farooq MSC C Clements PhD, Prof L Appleby FRCPsych, Prof N Kapur FR(Psych); Department of Psychiatry, Centre for Suicide Research, University of Oxford, Warneford Hospital, Oxford, UK (Prof K Hawton FMedsci, G Geulayov PhD, D Casey BSc); Oxford Health NHS Foundation Trust, Oxford, UK Prof K Hawton); Centre for Self harm and Suicide Prevention Research, Derbyshire Healthcare NHS Foundation Trust, Derby, UK (K Waters DipNurs, J Ness MSc, A Patel MSc, S Kelly PhD); School of Psychology, University of Nottingham, Nottingham, UK (Prof E Townsend PhD); Greater Manchester Mental Health NHS Foundation Trust, Manchester, UK (Prof N Kapur); The National Institute for Health Research Greater Manchester Patient Safety Translational Research Centre, Manchester, UK (Prof N Kapur)

Correspondence to: Miss Bushra Farooq, Centre for Mental Health and Safety, School of Health Sciences, Manchester Academic Health Science Centre, The University of Manchester, Manchester M13 9PL, UK bushra.farooq@manchester. ac.uk 
Research in context

\section{Evidence before this study}

Self-harm in young people is an increasing public health concern. Contemporary studies report increasing rates of self-harm in children and adolescents, but little is known about self-harm in children and adolescents from minority ethnic backgrounds who are known to have characteristics associated with poor outcomes. We search PubMed from Jan 1, 2000, to May 24, 2021, using search terms related to self-harm and mortality ("self-harm", "self-injury", "self-poisoning", "suicide attempt", "suicid*", "mortality", and "self-harm rates"); ethnicity ("ethnic minority", "Black", "Asian", " Pakistani", "Indian", "Chinese", "Bangladeshi", "mixed-race", and "mixed ethnicity"); and young people ("children", "adolescents", and "young people"). No language restrictions were applied. Studies relating to self-harm or mortality following self-harm by ethnic group were included, and excluded if ethnicity was not reported in the findings. One study investigated rates and repetition of selfharm in minority ethnic people older than 11 years and older than 65 years in London, UK. Compared with White people, Black and Asian people had lower rates of self-harm and 12-month repetition of self-harm across all age groups and sexes. However, people of mixed ethnicity had higher rates of repetition than White people, after adjustment for deprivation. Although several studies that investigated self-harm in minority ethnic people were identified, these were all done in adults. As of the cutoff date of our search (May 24, 2021), no studies had been published on the rates, characteristics, and outcomes of self-harm in children and adolescents from ethnic minorities.

\section{Added value of this study}

We used data from a large prospective study of hospital emergency department presentations for self-harm across five hospitals in three cities with varied sociodemographic profiles in England. The present study extends current understanding of self-harm in children and adolescents from under-represented and under-researched minority ethnic groups. Rates of self-harm were highest in White children and adolescents and increased between 2009 and 2016 in all ethnic groups, with slightly greater increases in rates of self-harm in minority groups. Black, South Asian, and other non-White children and adolescents were more likely to live in areas of high deprivation and were less likely to receive a specialist psychosocial assessment than White counterparts. Black and South Asian children and adolescents were also less likely to report a history of self-harm or to be receiving mental health care. There were proportionately more girls than boys in minority ethnic groups. Risk of repeat self-harm was lower in minority ethnic groups than for the White group in our study. However, there were no significant differences in suicide mortality between all groups.

Implications of all the available evidence

In our study, ethnic minority groups accounted for an increased proportion of self-harm, indicating the need for diverse preventive measures to reach all ethnic groups. Further research on the reasons for the increase in self-harm is also warranted. Integration of multi-agency support from schools, health and social care services, and families should address underlying mental health problems and socioeconomic disparities, and should promote positive mental health as preventive self-harm measures. Presentation to the emergency department is an important opportunity for intervention, and equitable access to a comprehensive psychosocial assessment needs to be ensured for all children and adolescents who self-harm. Any bias and discriminatory practices that are based on ethnicity also need to be identified and addressed to ensure effective management of self-harm in children and adolescents, in line with clinical guidance. as a coping mechanism, a strategy of emotion regulation, self-punishment, or a cry for help, among other things. ${ }^{8}$ Self-harm is associated with poor educational attainment and employment prospects, mental health problems, and increased risk of substance misuse in early adulthood., ${ }^{910}$

There is little research on the needs of young people from minority ethnic groups who self-harm. Socioeconomic disadvantage, parental unemployment, and living in lowincome and single-parent households are more common in young people from minority ethnic backgrounds; factors known to increase risk of self-harm in children and

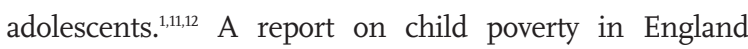
showed that more children of mixed, Black, or other (ie, Arab and other ethnicities) backgrounds were living in lowincome households than the national average, and Asian children were over two times more likely to live in persistently low-income household and socioeconomically deprived neighbourhoods. ${ }^{11,13}$ Socioeconomic deprivation is strongly associated with self-harm in young people. ${ }^{1}$
Rates of self-harm and all-cause mortality in minority ethnic adults are lower than those measured in White adults. ${ }^{14,15}$ Different ethnic and cultural identities, practices, beliefs, and senses of belonging might influence the meaning of self-harm and attitudes towards seeking help. ${ }^{15,16}$ Additionally, levels of social support and sense of community might vary between different cultural groups, as could stigma around seeking help from services and its effect on social status. ${ }^{17}$

Previous work on ethnicity and self-harm has largely been limited to adults and is dated. ${ }^{14}$ The COVID-19 pandemic has highlighted ethnic inequalities in health, and there is increasing emphasis on equity of access to mental health services and recognition of the need for further self-harm research to understand the needs of key marginalised groups for suicide and self-harm prevention. ${ }^{18}$ Those who present to hospital following selfharm might have more severe physical or psychological health needs and, because of their proximity to services, 
might be the group who are most amenable to intervention. ${ }^{19}$ Further work is needed to understand how people from minority ethnic backgrounds differ in characteristics and outcomes following self-harm, and to identify their needs and inform service provision.

We aimed to investigate self-harm that resulted in presentation to hospital in children and adolescents from minority ethnic groups compared with White children and adolescents. Additionally, we aimed to investigate rates of emergency department presentations for self-harm over time; to identify differences in clinical, demographic characteristics, measures of deprivation, self-harm methods, and problems precipitating self-harm; and to investigate repeat self-harm and mortality.

\section{Methods}

\section{Study design and participants}

Our observational cohort study included children and adolescents aged 10-19 years who presented to emergency departments following self-harm from Jan 1, 2000, to Dec 31, 2016, using data from the Multicentre Study of Self-harm in England. This ongoing, prospective study collects data for all hospital emergency department presentations for self-harm in five general hospitals in Manchester, Oxford, and Derby, in the UK. In the study, self-harm is defined as any act of intentional self-injury or self-poisoning, regardless of intent. Self-poisoning includes overdoses involving more than the prescribed amount of any drug, recreational drugs, non-ingestible substances, and instances of severe alcohol intoxication that are considered to be acts of self-harm by medical staff. Self-injury involves any type of intentional self-inflicted injury, regardless of medical severity.

In the present study, children and adolescents are defined as being aged 10-19 years, in line with the definition given by WHO, and were divided into three age bands based on periods of physical and psychological change: age 10-13 years (adjustment to secondary school and onset of self-harm); age 14-16 years (sharp increase in self-harm); and age 17-19 years (leaving school and transition into university or employment). ${ }^{1,20}$

The Multicentre Study of Self-harm in England was approved under Section 251 of the National Health Service (NHS) Act (2006) to collect patient-identifiable information without patient consent, and is compliant with the Data Protection Act (2018). The monitoring systems in Oxford and Derby have approval from local health research ethics committees. Self-harm monitoring in Manchester was part of a clinical audit system ratified by the local research ethics committee.

\section{Procedures}

Information on method of self-harm, sex, age, and ethnicity were collected for all self-harm presentations from emergency department databases and clinical hospital records. Detailed data (ie, previous self-harm, current and previous psychiatric care, problems precipitating self-harm, and aftercare offered to patients) were collected for people who received a specialist psychosocial assessment by psychiatry liaison staff (or an emergency department assessment in Manchester).

Given that ethnic groups were identified from information recorded in hospital records, the amount of detail for particular ethnic groups differed across study sites and over time. Groups were aggregated into broad categories: White (British, Welsh, Irish, Scottish, and any other White background), Black (African, Caribbean, and any other Black background), South Asian (Indian, Pakistani, Bangladeshi, and any other South Asian background), and other non-White (Arab, Chinese, mixed-ethnicity, and any other non-White background). Previous psychiatric care was defined as any care from secondary mental health services, and current psychiatric care encompassed current inpatient or outpatient care. Previous history of self-harm and problems precipitating self-harm were self-reported.

Census data from $2011^{21}$ showed that the largest minority ethnic group in the study areas (city of Manchester, Oxford city, Derby unitary area) was South Asian (12\%), followed by other non-White (8\%) and Black $(6 \%)$ groups. The proportions were slightly higher in the study areas than for England overall (South Asian: 7\%; other non-White: 4\%; and Black: 3\%). Manchester had higher proportions of ethnic minorities (South Asian: 14\%; other non-White: 10\%; and Black: 9\%) than Oxford (South Asian: 10\%; other non-White: 8\%; and Black: 5\%) and Derby (South Asian: 12\%; other non-White: and 5\%; Black: 3\%).

Index of Multiple Deprivation (IMD) scores were calculated using patients' postcodes. IMD measures smallarea deprivation on the basis of seven domains (income deprivation, employment deprivation, education, skills, and training deprivation, health deprivation and disability, crime, barriers to housing and services, and living environment deprivation). ${ }^{22}$ Areas in England were ranked from the most deprived to the least deprived on the basis of the average level of deprivation scores of neighborhoods within an area. IMD scores were aggregated into quintiles ranked from the least deprived in quintile 1 (IMD score $\leq 8.49$ ) to the most deprived in quintile 5 (IMD score $\geq 34 \cdot 18$ ). When no postcode was recorded, IMD scores were treated as missing. Manchester is the sixth most deprived local authority in England, Derby is ranked 67th, and Oxford is ranked 182nd.

Individuals were followed up for mortality through linkage with Office for National Statistics mortality records (via NHS Digital). Patient details such as name, date of birth, postcode, NHS number, and unique record identity numbers were used to link mortality data with the study self-harm dataset. Details for mortality linkage were taken from the first recorded self-harm presentation on the study database. Follow-up was until Dec 31, 2019, enabling a minimum of 3 years follow-up and a maximum of 20 years follow-up. Underlying cause of death was based
For more on the IMD tool see https://tools.npeu.ox.ac.uk/imd/

For more on the WHO definition of children and adolescents see https://www.who.int/healthtopics/adolescent-health 
on International Classification of Diseases-10 codes. Suicide included intentional self-harm (codes X60-X84) or death due to undetermined intent (Y10-Y34). ${ }^{23}$ Other causes included accidental deaths (V01-X59) and all other causes (natural deaths, codes R99 ill-defined or unspecified, and mental and behavioural disorders due to psychoactive substance use [F10-F19]).

\section{Statistical analysis}

Rates of self-harm between 2009 and 2016 by ethnic group (White, Black, South Asian, or other non-White) and sex were calculated using the first presentation for self-harm for each individual within each calendar year. Annual population estimates by ethnic group and single year of age were not available, and therefore data from the 2011 census were used to obtain population estimates for the study catchment areas by ethnic group. The study hospitals fell within the catchment areas of Oxford city, the city of Manchester, and Derby unitary area. Data for ethnicity before 2009 were less complete (ethnicity was missing for 2083 [28\%] of 7357 individuals); therefore, rates were calculated for the period 2009-16. Ethnicity data were available for 6632 (88\%) of 7536 individuals during this period. Poisson and negative binomial regression models (with exact $95 \%$ CIs) were used to examine trends over time in rates of self-harm. Pearson's goodness-of-fit tests assessed over-dispersion. Year (exposure) was entered as continuous variable in the Poisson model, and the rate of self-harm (per 100000 population) was the dependent variable.

Pearson's $\chi^{2}$ tests were used to investigate differences between ethnic groups in terms of demographic and clinical characteristics, methods of self-harm, and the problems that precipitated self-harm at patients' initial presentation for self-harm. Comparison of demographic characteristics (ie, age, sex, and level of deprivation) and method of self-harm included all patients, whereas comparison of clinical characteristics (history of previous

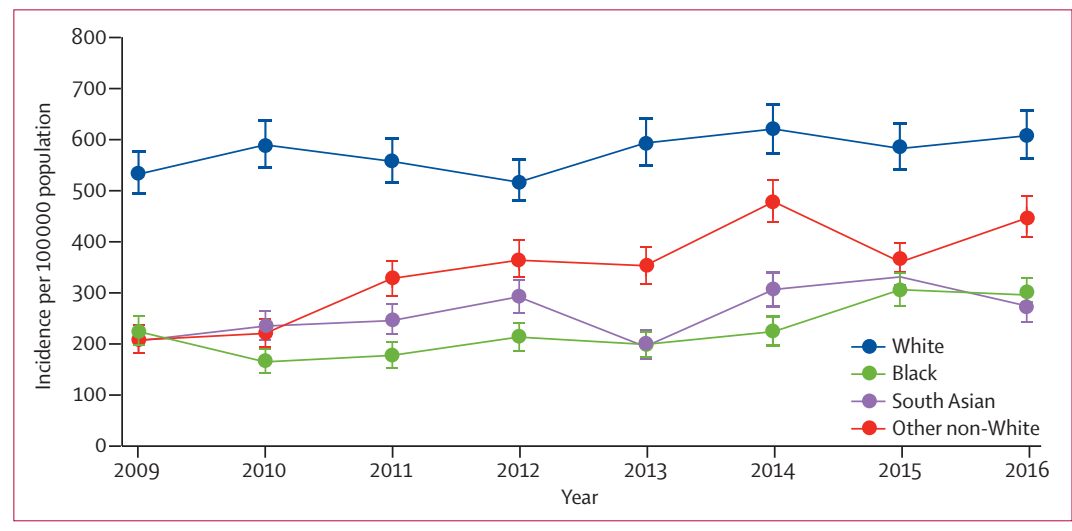

Figure 1: Self-harm per 100000 population in White, Black, South Asian, and other non-White young people aged $10-19$ years, $2000-16$

Error bars represent $95 \% \mathrm{Cls}$. Ethnic groups were aggregated into the following broad categories: White (British, Welsh, Irish, Scottish, and any other White background), Black (African, Caribbean, and any other Black background), South Asian (Indian, Pakistani, Bangladeshi, and any other South Asian background), and other nonWhite (Arab, Chinese, mixed-ethnicity, and any other non-White background) self-harm, current and previous psychiatric care, and alcohol use at the time of self-harm) and the problems that precipitated self-harm were restricted to people who received a specialist psychosocial assessment (or an emergency department assessment in Manchester) at their initial presentation. Only valid responses (yes or no) were included for each variable; people with missing information for specific factors were excluded from analyses of those variables.

12-month rates of repeat self-harm were calculated on the basis of re-presentation to study hospitals within 12 months of the first recorded presentation. Pearson's $\chi^{2}$ tests examined differences between the ethnic groups. Rates of self-harm repetition were calculated for individuals presenting up to Dec 31, 2015, to allow for 12-month follow-up.

Kaplan-Meier curves (with 95\% CIs) were plotted to compare time to first repeat self-harm presentation (from initial presentation) within 12 months between ethnic groups, and log-rank tests were used for comparison of group differences. Cox proportional hazards models generated hazard ratios (HRs) with $95 \%$ CIs to investigate the association between ethnicity and repetition of selfharm. An unadjusted model using the complete sample examined risk of 12-month repetition of self-harm between White (reference group) and Black, South Asian, and other non-White groups. An adjusted model (restricted to people who had a specialist psychosocial assessment or an emergency department assessment in Manchester) included multiple risk factors associated with repeat selfharm: age, sex, deprivation quintile, history of self-harm, and method of self-harm.?

Differences between ethnic groups in cause of death were compared using log-rank tests. Cox proportional hazards models generated HRs to compare risk of allcause mortality (from the initial presentation) between White (reference group) and Black, South Asian, and other non-White groups. Schoenfeld residuals and loglog plots of survival were used to test proportional hazards assumptions.

Sensitivity analyses used data from 2009 to 2016 because of the higher proportion of missing ethnicity data from 2000 to 2008 and to investigate any changes in demographic characteristics, clinical characteristics, and repetition of self-harm, when excluding data collected before 2009. Analyses were done in Stata, version 15.1 (StataCorp, College Station, TX, USA).

\section{Role of the funding source}

The funder of the study had no role in study design, data collection, data analysis, data interpretation, or writing of the report.

\section{Results}

There were 22128 hospital presentations for self-harm by 14894 individuals aged 10-19 years between Jan 1, 2000 and Dec 31, 2016. Of these presentations, $14189(75 \cdot 7 \%)$ 


\begin{tabular}{|c|c|c|c|c|c|c|}
\hline & $\begin{array}{l}\text { White children } \\
\text { and adolescents, } \\
\mathrm{n}=10211 \text { ( } \mathrm{n} \text { [valid \%]) }\end{array}$ & $\begin{array}{l}\text { Black children and } \\
\text { adolescents, } \mathrm{n}=344 \\
\text { (n [valid \%]) }\end{array}$ & $\begin{array}{l}\text { South Asian } \\
\text { children and } \\
\text { adolescents, n=619 } \\
\text { ( } \mathrm{n} \text { [valid \%]) }\end{array}$ & $\begin{array}{l}\text { Other non-white } \\
\text { children and } \\
\text { adolescents, n=732 } \\
\text { ( } \mathrm{n} \text { [valid \%]) }\end{array}$ & $\begin{array}{l}\text { Total, } \mathrm{n}=11906 \\
\text { (n [valid \%]) }\end{array}$ & $\chi^{2}$ test $^{*}$ \\
\hline \multicolumn{7}{|l|}{ Sex } \\
\hline Boys & $2813(27 \cdot 6 \%)$ & $69(20 \cdot 1 \%)$ & $122(19 \cdot 7 \%)$ & $179(24 \cdot 5 \%)$ & $3183(26 \cdot 7 \%)$ & $28.87(p<0.0001)$ \\
\hline Girls & $7396(72 \cdot 5 \%)$ & $275(79 \cdot 9 \%)$ & $497(80 \cdot 3 \%)$ & $553(75 \cdot 6 \%)$ & $8721(73 \cdot 3 \%)$ & $28.87(p<0.0001)$ \\
\hline Missing data (n [total \%]) & $2(<0 \cdot 1 \%)$ & $0(0.00 \%)$ & $0(0.00 \%)$ & $0(0.00 \%)$ & $2(<0 \cdot 1 \%)$ & NA \\
\hline \multicolumn{7}{|l|}{ Age group } \\
\hline $10-13$ years & $670(6 \cdot 6 \%)$ & $29(8 \cdot 4 \%)$ & $39(6 \cdot 3 \%)$ & $58(7 \cdot 9 \%)$ & $796(6 \cdot 7 \%)$ & $5.95(p=0.43)$ \\
\hline $14-16$ years & $3694(36 \cdot 2 \%)$ & $126(36.6 \%)$ & $215(34 \cdot 7 \%)$ & $275(37 \cdot 6 \%)$ & $4310(36 \cdot 2 \%)$ & $5.95(\mathrm{p}=0.43)$ \\
\hline $17-19$ years & $5847(57 \cdot 3 \%)$ & $189(54 \cdot 9 \%)$ & $365(59 \cdot 0 \%)$ & $399(54 \cdot 5 \%)$ & $6800(57 \cdot 1 \%)$ & $5.95(\mathrm{p}=0.43)$ \\
\hline \multicolumn{7}{|l|}{ Deprivation } \\
\hline Quintile 1 (IMD score $\leq 8 \cdot 49$; least deprived) & $1659(17 \cdot 2 \%)$ & $14(4 \cdot 3 \%)$ & $43(7 \cdot 5 \%)$ & $78(11 \cdot 2 \%)$ & $1794(16 \cdot 0 \%)$ & $261.28(p<0.0001)$ \\
\hline Quintile 2 (IMD score 8·5-13.79) & $1220(12 \cdot 7 \%)$ & $11(3 \cdot 4 \%)$ & $36(6 \cdot 3 \%)$ & $43(6 \cdot 2 \%)$ & $1310(11 \cdot 7 \%)$ & $261.28(p<0.0001)$ \\
\hline Quintile 3 (IMD score 13·80-21·35) & $1400(14 \cdot 5 \%)$ & $28(8.6 \%)$ & $75(13.0 \%)$ & $102(14 \cdot 6 \%)$ & $1605(14 \cdot 3 \%)$ & $261.28(p<0.0001)$ \\
\hline Quintile 4 (IMD score 21·36-34.17) & $1659(17 \cdot 2 \%)$ & $44(13 \cdot 5 \%)$ & $127(22 \cdot 1 \%)$ & $141(20 \cdot 2 \%)$ & $1971(17 \cdot 5 \%)$ & $261.28(p<0.0001)$ \\
\hline Quintile 5 (IMD score $\geq 34 \cdot 18$; most deprived) & $3705(38 \cdot 4 \%)$ & $229(70 \cdot 3 \%)$ & $294(51 \cdot 1 \%)$ & $334(47 \cdot 9 \%)$ & $4562(40 \cdot 6 \%)$ & $261.28(p<0.0001)$ \\
\hline Missing data (n [total \%]) & $568(5 \cdot 6 \%)$ & $18(5 \cdot 2 \%)$ & $44(7 \cdot 1 \%)$ & $34(4 \cdot 6 \%)$ & $664(5 \cdot 6 \%)$ & NA \\
\hline \multicolumn{7}{|l|}{ Clinical characteristics (method of self-harm) } \\
\hline Self-poisoning alone & $7457(73 \cdot 0 \%)$ & $293(85 \cdot 2 \%)$ & $512(82 \cdot 7 \%)$ & $532(72 \cdot 7 \%)$ & $8794(73.9 \%)$ & $56.06(p<0.0001)$ \\
\hline Self-injury alone & $2082(20 \cdot 4 \%)$ & $43(12 \cdot 5 \%)$ & $88(14 \cdot 2 \%)$ & $158(21 \cdot 6 \%)$ & $2371(19 \cdot 9 \%)$ & $56.06(p<0.0001)$ \\
\hline Both self-poisoning and self-injury & $672(6.6 \%)$ & $8(2 \cdot 3 \%)$ & $19(3 \cdot 1 \%)$ & $42(5 \cdot 7 \%)$ & $741(6 \cdot 2 \%)$ & $56.06(p<0.0001)$ \\
\hline \multicolumn{7}{|l|}{ Assessment, history, and psychiatric care } \\
\hline Specialist psychosocial assessment & $6679(65 \cdot 4 \%)$ & $179(52 \cdot 0 \%)$ & $330(53 \cdot 3 \%)$ & $405(55 \cdot 3 \%)$ & $7593(63 \cdot 8 \%)$ & $84.57(p<0.0001)$ \\
\hline $\begin{array}{l}\text { Emergency department assessment only (in } \\
\text { Manchester) }\end{array}$ & $1184(11 \cdot 6 \%)$ & $71(20 \cdot 6 \%)$ & $106(17 \cdot 1 \%)$ & $66(9 \cdot 0 \%)$ & $1427(12 \cdot 0 \%)$ & $47.47(p<0.0001)$ \\
\hline Total assessed & $7863(77 \cdot 0 \%)$ & $250(72 \cdot 7 \%)$ & $436(70 \cdot 4 \%)$ & $471(64 \cdot 3 \%)$ & $9020(75 \cdot 8 \%)$ & NA \\
\hline History of previous self-harm $\dagger(\mathrm{n}[\%])$ & $3800(55 \cdot 3 \%) ; n=6877$ & $106(46 \cdot 5 \%) ; n=228$ & $176(44 \cdot 6 \%) ; n=395$ & $225(55 \cdot 8 \%) ; \mathrm{n}=403$ & $4307(54 \cdot 5 \%) ; n=7903$ & $23.52(p<0.0001)$ \\
\hline Previous psychiatric care† (n [\%]) & $2776(38 \cdot 4 \%) ; n=7221$ & $63(26 \cdot 7 \%) ; n=236$ & $91(23 \cdot 1 \%) ; n=394$ & $154(35 \cdot 6 \%) ; n=433$ & $3084(37 \cdot 2 \%) ; n=8284$ & $49.95(p<0.0001)$ \\
\hline Current psychiatric caret (n [\%]) & $1289(18 \cdot 2 \%) ; n=7081$ & $22(9 \cdot 9 \%) ; n=222$ & $42(10 \cdot 6 \%) ; n=396$ & $71(17 \cdot 0 \%) ; n=418$ & $1424(17 \cdot 5 \%) ; n=8117$ & $24.34(p<0.0001)$ \\
\hline $\begin{array}{l}\text { Alcohol involved at the time of self-harm } \dagger \\
(\mathrm{n}[\%])\end{array}$ & $2378(34 \cdot 5 \%) ; n=6903$ & $53(24 \cdot 3 \%) ; n=218$ & $40(11 \cdot 0 \%) ; n=365$ & $106(26 \cdot 7 \%) ; n=397$ & $2577(32 \cdot 7 \%) ; n=7883$ & $101.47(p<0.0001)$ \\
\hline $\begin{array}{l}\text { Valid } \%=\% \text { of observations for each variable when } \\
{ }^{*} \chi^{2} \text { test for overall association of multicategorical } \\
736(8.2 \%) \text { for previous psychiatric care, } n=657 \text { (7) }\end{array}$ & 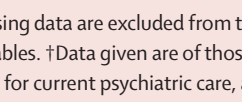 & Talo or & revith a & at the time of self-har & 西 & $\begin{array}{l}\text { n. NA=not applicable. } \\
\text { r history of self-harm, }\end{array}$ \\
\hline
\end{tabular}

were by females, and 11154 (59 - 5\%) were aged 17-19 years. Data for ethnicity were available for 18734 (84.7\%) of 22128 presentations for 11906 (79.9\%) of 14894 individuals. $10211(85.8 \%)$ of 11906 presenting individuals were White, $344(2 \cdot 9 \%)$ were Black, 619 (5.2\%) were South Asian, and $732(6 \cdot 1 \%)$ were other non-White. Overall, children and adolescents of any ethnic minority background accounted for $2306(12 \cdot 3 \%)$ of 18734 presentations within the study sample; $454(19.7 \%)$ of these presentations were by Black people, 849 (36.8\%) were by South Asian people, and $1003(43 \cdot 5 \%)$ were by other non-White people.

Rates of self-harm presenting to hospital were higher in White people across the study period (figure 1). Increases in rates of self-harm were observed from 2009 to 2016 in all ethnic groups. The increase appeared slightly greater in Black (incidence rate ratio 1.07 [95\% CI 1.03-1.11], $\mathrm{p}=0 \cdot 0005)$, South Asian (1.05 [1.01-1.09], $\mathrm{p}=0.026)$ and other non-White children and adolescents $(1.11$
[1.06-1.16], $\mathrm{p}<0 \cdot 0001)$ than in White children and adolescents (1.02 [1.00-1.03], $\mathrm{p}=0 \cdot 012)$. The models suggested an annual increase of $2 \%$ in the rates of selfharm in White, 7\% in Black, 5\% in South Asian, and 11\% in other non-White groups. Greater increases in rates of self-harm were observed from 2014 onwards in other nonWhite, Black, and South Asian groups, although rates of self-harm in the minority ethnic groups were based on smaller numbers of presentations relative to rates calculated for White groups.

Between 2009 and 2016, mean annual rates of self-harm per 100000 population were 574 for White, 225 for Black, 260 for South Asian, and 344 for other non-White children and adolescents. Mean annual rates of self-harm for boys were 304 per 100000 population in White children and adolescents, 97 in Black children and adolescents, 85 in South Asian children and adolescents, and 182 in other non-White children and adolescents. Rates of self-harm 


\begin{tabular}{|c|c|c|c|c|c|}
\hline & $\begin{array}{l}\text { White children and } \\
\text { adolescents, } \mathrm{n}=7863 \\
\text { ( } \mathrm{n}[\mathrm{valid} \%] \text { ) }\end{array}$ & $\begin{array}{l}\text { Black children and } \\
\text { adolescents, } n=250 \\
\text { ( } \mathrm{n}[\text { valid \%]) }\end{array}$ & $\begin{array}{l}\text { South Asian children and } \\
\text { adolescents, } \mathrm{n}=436 \\
\text { (n [valid \%]) }\end{array}$ & $\begin{array}{l}\text { Other non-white children } \\
\text { and adolescents, } \mathrm{n}=471 \\
\text { ( } \mathrm{n} \text { [valid \%]) }\end{array}$ & $\chi^{2}$ test \\
\hline Relationship problems with a partner & $2461(32 \cdot 6 \%) ; n=7555$ & $50(21 \cdot 7 \%) ; n=231$ & $120(29 \cdot 3 \%) ; n=410$ & $116(25 \cdot 6 \%) ; n=454$ & $22.47(p<0.0001)$ \\
\hline Relationship problems with family & $3258(43 \cdot 1 \%) ; n=7563$ & $124(53 \cdot 5 \%) ; n=232$ & $217(52 \cdot 9 \%) ; n=410$ & $214(47 \cdot 0 \%) ; n=455$ & $25.97(p<0.0001)$ \\
\hline Relationship problems with others & $1335(17 \cdot 7 \%) ; n=7560$ & $34(14 \cdot 7 \%) ; n=232$ & $55(13 \cdot 4 \%) ; n=410$ & $84(18 \cdot 5 \%) ; n=455$ & $6.45(p=0.092)$ \\
\hline Employment or study problems & $1760(23 \cdot 3 \%) ; n=7561$ & $58(25 \cdot 0 \%) ; n=232$ & $102(24 \cdot 9 \%) ; n=410$ & $128(28 \cdot 1 \%) ; n=455$ & $6 \cdot 22(p=0 \cdot 10)$ \\
\hline Financial problems & $512(6 \cdot 8 \%) ; n=7560$ & $17(7 \cdot 3 \%) ; n=232$ & $13(3 \cdot 2 \%) ; n=410$ & $30(6.6 \%) ; n=455$ & $8.38(p=0.039)$ \\
\hline Housing problems & $606(8 \cdot 0 \%) ; n=7561$ & $26(11 \cdot 2 \%) ; n=232$ & $14(3 \cdot 4 \%) ; n=410$ & $38(8 \cdot 4 \%) ; n=455$ & $15 \cdot 09(p=0 \cdot 0017)$ \\
\hline Legal problems & $172(2 \cdot 3 \%) ; n=7561$ & $7(3 \cdot 0 \%) ; n=232$ & $10(2 \cdot 4 \%) ; n=410$ & $16(3 \cdot 5 \%) ; n=455$ & $3 \cdot 31(p=0 \cdot 35)$ \\
\hline Drug or alcohol problems & $901(15 \cdot 1 \%) ; n=5953$ & $21(13 \cdot 2 \%) ; n=159$ & $13(4 \cdot 4 \%) ; n=297$ & $33(8.9 \%) ; n=373$ & $36.51(p<0.0001)$ \\
\hline Physical health problems & $317(4 \cdot 2 \%) ; n=7561$ & $10(4 \cdot 3 \%) ; n=232$ & $13(3 \cdot 2 \%) ; n=410$ & $24(5 \cdot 3 \%) ; n=455$ & $2.39(p=0.49)$ \\
\hline Mental health problems & $1366(18 \cdot 1 \%) ; n=7559$ & $33(14 \cdot 2 \%) ; n=232$ & $51(12 \cdot 4 \%) ; n=410$ & $93(20 \cdot 4 \%) ; n=455$ & $12 \cdot 61(p=0.0056)$ \\
\hline Bereavement & $571(7 \cdot 7 \%) ; n=7423$ & $15(6 \cdot 5 \%) ; n=231$ & $12(3.0 \%) ; n=403$ & $34(7 \cdot 6 \%) ; n=445$ & $12.68(p=0.0054)$ \\
\hline Abuse (physical, sexual, or emotional) & $802(10 \cdot 3 \%) ; n=7555$ & $21(9 \cdot 1 \%) ; n=231$ & $38(9 \cdot 3 \%) ; n=410$ & $52(11 \cdot 4 \%) ; n=455$ & $1.64(p=0.65)$ \\
\hline Any other problem & $1591(21 \cdot 2 \%) ; n=7521$ & $43(18 \cdot 6 \%) ; n=231$ & $85(20 \cdot 7 \%) ; n=410$ & $115(25 \cdot 4 \%) ; n=452$ & $5.80(p=0.12)$ \\
\hline
\end{tabular}

Table 2: Comparison of problems that precipitated self-harm

\begin{tabular}{|c|c|c|c|c|c|}
\hline & $\begin{array}{l}\text { Repeat self-harm in children and adolescents } \\
\text { with complete 12-month follow-up }(n=1980) \text {, } \\
\text { n (valid \%) }\end{array}$ & $\begin{array}{l}\text { Crude hazard } \\
\text { ratio }(95 \% \mathrm{Cl})\end{array}$ & $p$ value & $\begin{array}{l}\text { Adjusted hazard } \\
\text { ratio }(95 \% \mathrm{Cl})\end{array}$ & $\mathrm{p}$ value \\
\hline $\begin{array}{l}\text { White children and adolescents } \\
\text { (reference group) }\end{array}$ & $1763(18.8 \%) ; n=9395$ & .. & .. & .. & .. \\
\hline Black children and adolescents & $46(14 \cdot 6 \%) ; n=315$ & $0.73(0.54-0.97)$ & $p=0.028$ & $0.64(0.42-0.97)^{*}$ & $p=0.034$ \\
\hline South Asian children and adolescents & $78(13.7 \%) ; n=571$ & $0.69(0.56-0.87)$ & $p=0.0011$ & $0.85(0.63-1.14)^{*}$ & $\mathrm{p}=0.28$ \\
\hline $\begin{array}{l}\text { Other non-white children and } \\
\text { adolescents }\end{array}$ & $93(14 \cdot 3 \%) ; n=652$ & $0.72(0.59-0.88)$ & $p=0.0013$ & $0.82(0.62-1.08)^{*}$ & $p=0.15$ \\
\hline \multicolumn{6}{|c|}{$\begin{array}{l}\text { Valid } \%=\% \text { of observations for each variable when missing data are excluded from the total. * Restricted to assessed presentations and adjusted for age, sex, level of } \\
\text { deprivation, previous self-harm, and method of self-harm. }\end{array}$} \\
\hline
\end{tabular}

were higher in girls than for boys across all ethnicities; 848 per 100000 population in White children and adolescents, 353 in Black, 369 in South Asian, and 659 in other non-White children and adolescents.

A higher proportion of girls than boys presented with self-harm across all ethnic groups (table 1). This See Online for appendix proportion was slightly higher in the South Asian group (497 [80 - 3\%] of 619) than in the White group (7396 [72 -5\%] of 10211).

Black, South Asian, and other non-White children and adolescents were more likely to live in areas of high deprivation than White children and adolescents (table 1). The proportion of Black children and adolescents in the most deprived quintile was higher than all other groups.

Of those who had an assessment, history of self-harm was more common in children and adolescents from other non-White (225 [55.8\%] of 403 with data) and White (3800 [55.3\%] of 6877 with data) ethnic groups than those from Black (106 [46 · 5\%] of 228 with data) and South Asian (176 [44.6\%] of 395 with data) ethnic groups ( $<<0 \cdot 0001$; table 1). White and other non-White ethnic groups more often received current psychiatric care. Black (179 [52 .0\%] of 344), South Asian (330 [53.3\%] of 619), and other
non-White (405 [55 -3\%] of 732) children and adolescents were less likely to receive a specialist psychosocial assessment than White children and adolescents (6679 [65.4\%] of $10208 ; \mathrm{p}<0 \cdot 0001$ ). Details of a post-hoc analysis of the differences in assessments between children and adolescents aged 10-15 years and adolescents aged 16-19 years are in the appendix (p 1). The analysis showed the difference in psychosocial assessment between White and minority ethnic groups was greater in children and adolescents aged 10-15 years (those who may be assessed and treated by child and adolescent mental health services) than in those aged 16-19 years (assessed and treated by adult liaison services).

Self-poisoning was the most common method of selfharm across all groups (table 1). About a quarter of Black (53 [24.3\%] of 218) and other non-White (106 [26.7\%] of 397) children and adolescents consumed alcohol at the time of self-harm, compared with 2378) 34.5\%) of 6903 White and $40(11.0 \%)$ of 365 South Asian children and adolescents $(\mathrm{p}<0 \cdot 0001)$.

Relationship problems with family was the most commonly reported precipitant of self-harm in all ethnic groups. However, a larger proportion of South Asian and 
Black children and adolescents reported relationship problems with family than other ethnic groups (table 2). White children and adolescents more often reported relationship problems with a partner at the time of selfharm. Black children and adolescents more often reported housing and financial problems than did other groups, and other non-White children and adolescents more often reported mental health problems. White and Black children and adolescents more often reported problems with drugs and alcohol compared with other groups. Abuse (physical, sexual, or emotional) was a commonly reported precipitant of self-harm in all ethnic groups (table 2).

$10933(91 \cdot 8 \%)$ of 11906 children and adolescents had a complete 12-month follow-up, of whom 1980 (18.1\%) had repeat self-harm within 12-months (table 3). Repeat self-harm was highest in White children and adolescents (1763 [18.8\%] of 9395), compared with Black (46 [14.6\%] of 315), South Asian (78 [13.7\%] of 571), and other non-White ethnic groups (93 [14.3\%] of 652; $\mathrm{p}=0 \cdot 0002$ ). Log-rank tests of the Kaplan-Meier curves showed that the time to repeat self-harm was significantly different $(p=0 \cdot 0002)$ between groups, with White children and adolescents repeating self-harm more rapidly (figure 2).

Results from an unadjusted Cox proportional hazards model showed lower risk of repeat self-harm in Black (HR 0.73 [95\% CI 0.54-0.97], $\mathrm{p}=0.028$ ), South Asian (0.69 [0.56-0.87], $\mathrm{p}=0.0011)$, and other non-White groups $(0 \cdot 72[0 \cdot 59-0 \cdot 88], \mathrm{p}=0 \cdot 0013)$ than White children and adolescents (table 3 ). In an adjusted Cox proportional hazards model of 7497 individuals, lower risk of repeat self-harm only maintained significance in Black children and adolescents $(0 \cdot 64[0 \cdot 42-0 \cdot 97], p=0 \cdot 034)$. In the fully adjusted model (appendix p 2), those with a history of self-harm were twice as likely to repeat self-harm, and high deprivation was also associated with increased risk across all groups.

Mortality follow-up data were available for 11108 (93 - 3\%) of 11906 individuals; $10753(96.8 \%)$ were alive, 192 (1.7\%) had died, and 163 (1.5\%) had emigrated and their status was not known. Deaths were reported in $178(1.9 \%)$ of 9432 White, four $(1.4 \%)$ of 295 Black, six $(1.1 \%)$ of 571 South Asian, and four ( $0 \cdot 6 \%)$ of 647 other non-White children and adolescents.

Among the 192 children and adolescents who died, $77(40 \cdot 1 \%)$ died by suicide, $51(26 \cdot 7 \%)$ were accidental deaths, and $64(33 \cdot 3 \%)$ died from other causes (ie, natural causes, ill-defined or unspecified causes, and mental and behavioural disorders due to psychoactive substance use). $72(40.4 \%)$ of 178 deaths in White children and adolescents and $5(35.7 \%)$ of 14 deaths in Black, South Asian, and other non-White children and adolescents were by suicide (figures by ethnic subgroups omitted because of small numbers). Log-rank tests showed no significant differences in all-cause mortality $(\mathrm{p}=0 \cdot 14)$, accidental mortality $(\mathrm{p}=0 \cdot 72)$, or suicide $(\mathrm{p}=0 \cdot 32)$ mortality between ethnic

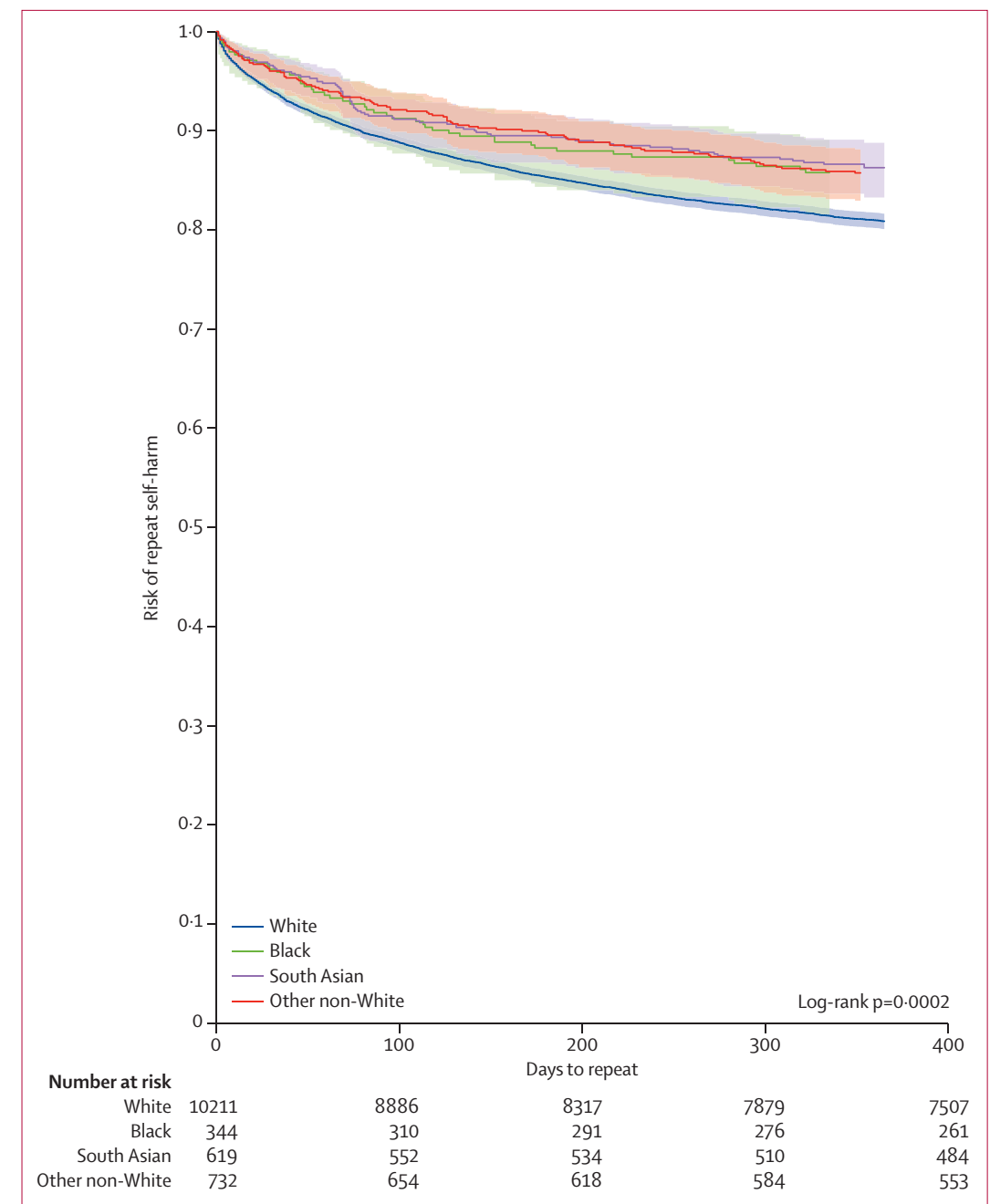

Figure 2: Kaplan-Meier curve for risk of 12-month repeated self-harm in children and adolescents by ethnic group

Width of shaded area represents $95 \%$ Cls. Ethnic groups were aggregated into the following broad categories: White (British, Welsh, Irish, Scottish, and any other White background), Black (African, Caribbean, and any other Black background), South Asian (Indian, Pakistani, Bangladeshi, and any other South Asian background), and other non-White (Arab, Chinese, mixed-ethnicity, and any other non-White background)

groups. In unadjusted Cox regression models, there were no statistically significant differences in HRs for all-cause mortality in Black (HR 0 70 [95\% CI 0.26-1.87], $\mathrm{p}=0 \cdot 47$ ), South Asian $(0 \cdot 58$ [0.26-1.31], $\mathrm{p}=0 \cdot 19)$, and other nonWhite children and adolescents $(0 \cdot 40$ [0.15-1.09], $\mathrm{p}=0.073$ ) compared with White children and adolescents. In a post-hoc analysis, compared with White children and adolescents, children and adolescents from all other minority ethnic groups combined showed a lower risk of mortality $(\operatorname{HR} 0 \cdot 54$ [0.31-0.93], $\mathrm{p}=0 \cdot 026)$.

Sensitivity analyses using data from 2009 to 2016 showed that the associations between ethnic group and demographic and clinical characteristics, methods of selfharm, problems precipitating self-harm, and rates of repeat self-harm were broadly similar to those based on the full sample. However, the difference in the 12-month 
risk of repeat self-harm between White and Black ethnic groups in the Cox proportional hazards models was no longer significant, perhaps because of the relatively small size of the Black group.

\section{Discussion}

This study investigated hospital-presenting self-harm, repeat self-harm, and mortality in children and adolescents across ethnic groups using a large multisite dataset. Rates of self-harm were higher in White children and adolescents than in minority ethnic people; however, increases in rates of self-harm over time appeared to be somewhat greater in Black, South Asian, and other non-White groups. There were proportionately more girls than boys in ethnic minority groups than in White groups, especially in the South Asian group. Black children and adolescents accounted for the largest proportion of those living in the most deprived areas.

South Asian and Black children and adolescents were less likely to report a history of self-harm or to be in receipt of psychiatric care, and more often reported relationship problems with family than did other groups. Alcohol use at the time of self-harm was less common in South Asian children and adolescents. Abuse was reported as precipitating self-harm by around $10 \%$ of children and adolescents in all groups.

Black, South Asian, and other non-White children and adolescents were less likely to receive a specialist psychosocial assessment than White children and adolescents. These groups also had lower rates of repeat self-harm; however, this association was attenuated after adjusting for known risk factors. There was no difference between ethnic groups in suicide mortality. However, numbers were small and should be interpreted with caution.

The increases in rates of self-harm over time was consistent with previous work on presentations for selfharm to general practice and to hospital emergency departments. ${ }^{1,24}$ Greater increases in self-harm in minority ethnic groups could partly be explained by improved recording of ethnicity over time. However, although there was a trend towards increased recording of ethnicity across the study period, completion was relatively stable from 2009 to 2016 and this factor was unlikely to account for the rapid increase in rates.

Increases in self-harm across ethnic groups could reflect the emergence of mental health problems during adolescence and increasing mental health distress, perhaps driven by increased distress and internalising of symptoms in girls found in more recent cohorts in England. ${ }^{25-27}$ Rapid social change since 2010 might also have had a role, including the prevalence of social media use, cyberbullying, negative body image, low self-esteem, and increases in depressive symptoms. ${ }^{28-30}$ Increases in help-seeking for self-harm could have also contributed to the observed increase.$^{31}$ Increases in the rates of self-harm after 2011 could be associated with deprivation, poverty, and reductions in young people's mental health services as a consequence of austerity measures following the 2008 economic recession. These changes most affected those living in poorer communities, which include a disproportionate number of minority ethnic groups. ${ }^{32}$

Minority ethnic children and adolescents in this study were socioeconomically disadvantaged. The highest proportion in the most socioeconomically deprived quintile were Black children and adolescents. This group was less likely to receive a specialist psychosocial assessment, to be in receipt of mental health care, or to report a history of self-harm than were White ethnic groups. ${ }^{14}$ Lower proportions of Black and South Asian children and adolescents in receipt of mental health care could reflect the under-representation and lower service use rates of minority ethnic groups in child and adolescent mental health services. ${ }^{1,33}$ Ethnic and cultural differences in help-seeking, self-reliance, and the relevance of mainstream services in meeting the needs of minority ethnic groups could also explain the disparities in mental health care. ${ }^{17}$

The lower risk of 12-month repeat self-harm in minority ethnic groups was consistent with findings from minority ethnic adults. ${ }^{7,16}$ Those with a history of self-harm were twice as likely to repeat self-harm, ${ }^{7}$ and higher levels of deprivation were associated with an increase in repeat self-harm in the fully-adjusted Cox regression model. Children and adolescents from socioeconomically deprived areas were less likely to receive follow-up care than were those from less deprived areas, and subsequently can have poorer outcomes. ${ }^{1}$ By contrast with previous work ${ }^{15}$ that showed that Black and South Asian adults were at a lower risk of suicide than White people, there was no difference in suicide mortality in our study.

A large dataset and long follow-up is a strength of this study, allowing for exploration of trends over time. Due to the unavailability of annual population-level data for ethnicity, data from the 2011 census were used as the denominator for rates of self-harm and might not have reflected changes in ethnic minority populations due to migration or other causes. If ethnic minority populations in the study areas increased more rapidly than White populations did in recent years, using 2011 census data as the denominator for these later years would have artificially inflated calculated rates for ethnic minority groups. The size of ethnic minority groups in our study (especially for Black children and adolescents) were also small relative to those of White children and adolescents, meaning that rates of self-harm need to be interpreted with caution. As our data were limited to three largely urban areas, we were not able to examine differences in self-harm between urban and rural areas. Therefore, our findings might not be representative of ethnic minority groups in rural areas in England.

Analyses of clinical characteristics and problems precipitating self-harm were based on assessed individuals 
only. Children and adolescents who did not receive a specialist psychosocial assessment might have differed in their characteristics and outcomes. Repeat presentations for self-harm made to hospitals outside of the study areas could not be captured; therefore, rates and risk of repeat self-harm might have been underestimated. However, previous audits ${ }^{34}$ have found that the Multicentre Study of Self-harm has complete coverage of the cities included in our study and captured most emergency department presentations for self-harm by residents in the study areas.

We used aggregated categories of ethnicity in our study, meaning that important differences in characteristics and outcomes in each minority ethnic subgroup could not be explored. However, it was not possible to disaggregate these groups in this study because of differences in recording across study sites. Cultural factors that influence self-harm and help-seeking could not be examined. People from diverse ethnic groups might respond to distress in different ways and attitudes to helpseeking are influenced by their beliefs about self-harm. ${ }^{17}$

Our study is based on hospital presentations for selfharm, whereas self-harm trends, characteristics, and outcomes in community settings might differ. However, self-harm in community settings was beyond the scope of this study.

Self-harm in children and adolescents has become a major public health concern in recent years and minority ethnic groups appeared to account for an increasing proportion of self-harm episodes. Further understanding of the psychosocial factors behind this increase is required, and effective preventive measures must be designed to reach all ethnic groups.

Children and adolescents from minority backgrounds were from deprived communities and more often reported relationship problems with family. Other non-White groups more often reported a history of self-harm and were more likely to have been in receipt of psychiatric care than were other ethnic groups. There were some factors (eg, adverse experiences related to physical, sexual, or emotional abuse) that were reported by all ethnic groups. Integral to the prevention of self-harm is the promotion of positive mental health and the integration of accessible support from health and social care services, families, and schools to address underlying mental health problems, social problems, and major trauma (eg, physical, sexual, or emotional abuse).

Services designed for minority ethnic people delivered in community settings and schools are feasible, known to have better engagement, and improve mental health outcomes compared with mainstream services. ${ }^{35}$ Culturally adapted resources and psychoeducation on mental health and self-harm for parents and carers to increase knowledge and reduce stigma, as well as outreach programmes in local community health centres to support socioeconomically disadvantaged families could be helpful for reducing self-harm in ethnic minority children and adolescents. Further research on the efficacy of culturally adapted interventions for children and adolescents is needed.

Approximately 200000 children and adolescents self-harm in community settings; therefore, specific prevention and intervention is important to reduce the burden of self-harm outside of hospital settings. ${ }^{2}$ Promotion of positive mental health and wellbeing, with a focus on family, relationships, and psychosocial functioning, are also essential to prevent self-harm and poor mental health outcomes. School-based suicide prevention programmes aimed at raising mental health awareness and developing coping skills, have been effective at reducing suicide attempts and ideation in children and adolescents. ${ }^{36}$

Presentation to hospital is an opportunity for intervention and equitable access to services should be prioritised. Clinical guidance recommends that all those who present to hospital for self-harm should receive a comprehensive psychosocial assessment. ${ }^{37}$ However, children and adolescents from minority ethnic backgrounds were less likely to receive an assessment. Examination of psychosocial assessment by age group (people under 16 years vs people aged 16-19 years) showed that ethnic minorities across both age groups were less likely to be assessed than White groups. The difference in rates of assessment might have been even larger in younger age groups, although further exploration is needed. Although easily accessible and culturally-sensitive mental health services are central to ensuring equity in service provision for ethnic minority communities, we also need to ensure that services are fit for purpose when young people present. Bias among staff-unconscious or conscious-and discriminatory practices within services also need to be identified and addressed to ensure that children and adolescents who self-harm are effectively managed in line with clinical guidance. Training and support for psychiatric liaison staff on cultural competence and ethnic diversity also needs to be ensured.

\section{Contributors}

$\mathrm{BF}, \mathrm{CC}$, and NK were responsible for the study conception and design. $\mathrm{BF}$ was the guarantor of the study and was responsible for data analysis and writing of the initial draft. BF also had full access to all the data in the study and accepted responsibility to submit for publication. CC and NK were responsible for review and editing. All authors had access to the data (CC and GG verified the data) and were involved in interpretation of the results, critical review of the manuscript, and approval of the final draft.

\section{Declaration of interests}

LA chairs the National Suicide Prevention Strategy Advisory Group of the UK Department of Health and Social Care, which NK and $\mathrm{KH}$ are members of. NK reports grants from Department of Health and Social Care, National Institute of Health Research, National Institute of Health and Care Excellence, and Healthcare Quality and Improvement Partnership, outside of the submitted work. NK has chaired the National Institute for Health and Care Excellence (NICE) guidelines for the longer-term management of self-harm, is the clinical topic adviser for the new NICE self-harm guidelines, chairs the guideline committee for the management of depression, and is part of the NICE Topic Expert Group, which developed the quality standards for self-harm services. $\mathrm{KW}$ is executive director for STORM UK suicide prevention training. All other authors declare no competing interests. 


\section{Data sharing}

Individual patient-level will not be available because of confidentiality and data-sharing agreements in place. The study protocol, statistical analysis plan, and analytic code is available on request from the corresponding author.

\section{Acknowledgments}

We would like to thank Elizabeth Bale, Fiona Brand, and members of the clinical service in Oxford and data collection and processing teams in Manchester and Derbyshire Healthcare Foundation Trust.

References

1 Morgan C, Webb RT, Carr MJ, et al. Incidence, clinical management, and mortality risk following self harm among children and adolescents: cohort study in primary care. BMJ 2017; 359: j4351.

2 Geulayov G, Casey D, McDonald KC, et al. Incidence of suicide, hospital-presenting non-fatal self-harm, and community-occurring non-fatal self-harm in adolescents in England (the iceberg model of self-harm): a retrospective study. Lancet Psychiatry 2018; 5: 167-74.

3 Patalay P, Gage SH. Changes in millennial adolescent menta health and health-related behaviours over 10 years: a population cohort comparison study. Int J Epidemiol 2019; 48: 1650-64.

4 Department of Health and Social Care. Preventing suicide in England: a cross-government outcomes strategy to save lives. 2012. https://assets.publishing.service.gov.uk/government/uploads/ system/uploads/attachment_data/file/430720/Preventing-Suicide-.pdf (accessed Aug 8, 2021).

5 Hawton K, Bale L, Brand F, et al. Mortality in children and adolescents following presentation to hospital after non-fatal self-harm in the Multicentre Study of Self-harm: a prospective observational cohort study. Lancet Child Adolesc Health 2020; 4: 111-20.

6 Hawton K, Bergen H, Waters K, et al. Epidemiology and nature of self-harm in children and adolescents: findings from the multicentre study of self-harm in England. Eur Child Adolesc Psychiatry 2012; 21: $369-77$.

7 Hawton K, Bergen H, Kapur N, et al. Repetition of self-harm and suicide following self-harm in children and adolescents: findings from the Multicentre Study of Self-harm in England. J Child Psychol Psychiatry 2012; 53: 1212-19.

8 Scoliers G, Portzky G, Madge N, et al. Reasons for adolescent deliberate self-harm: a cry of pain and/or a cry for help? Findings from the child and adolescent self-harm in Europe (CASE) study. Soc Psychiatry Psychiatr Epidemiol 2009; 44: 601-07.

9 Mars B, Heron J, Klonsky ED, et al. Predictors of future suicide attempt among adolescents with suicidal thoughts or non-suicida self-harm: a population-based birth cohort study. Lancet Psychiatry 2019; 6: 327-37.

10 Mars B, Heron J, Crane C, et al. Clinical and social outcomes of adolescent self harm: population based birth cohort study. BMJ 2014; 349: g5954.

11 Office for National Statistics. Child poverty and education outcomes by ethnicity. 2020. https://www.ons.gov.uk/economy/ nationalaccounts/uksectoraccounts/compendium/economicreview february2020/childpovertyandeducationoutcomesbyethnicity (accessed July 23, 2020).

12 Mok PLH, Antonsen S, Pedersen CB, et al. Family income inequalities and trajectories through childhood and self-harm and violence in young adults: a population-based, nested case-control study. Lancet Public Health 2018; 3: e498-507.

13 Ministry of Housing, Communities and Local Government. People living in deprived neighbourhoods. 2020. https://www.ethnicityfacts-figures.service.gov.uk/uk-population-by-ethnicity/ demographics/people-living-in-deprived-neighbourhoods/ latest\#main-facts-and-figures (accessed July 24, 2020).

14 Cooper J, Murphy E, Webb R, et al. Ethnic differences in self-harm, rates, characteristics and service provision: three-city cohort study. Br J Psychiatry 2010; 197: 212-18.

15 Turnbull P, Webb R, Kapur N, et al. Variation by ethnic group in premature mortality risk following self-harm: a multicentre cohort study in England. BMC Psychiatry 2015; 15: 254.

16 Polling C, Bakolis I, Hotopf M, Hatch SL. Variation in rates of self-harm hospital admission and re-admission by ethnicity in London: a population cohort study. Soc Psychiatry Psychiatr Epidemiol 2021; published online April 20. https://doi.org/10.1007/s00127-02102087-9.
17 Polling C, Woodhead C, Harwood H, Hotopf M, Hatch SL. "There is so much more for us to lose if we were to kill ourselves": understanding paradoxically low rates of self-harm in a socioeconomically disadvantaged community in London. Qual Health Res 2021; 31: 122-36.

18 Department of Health and Social Care. Preventing suicide in England: fifth progress report of the cross government outcomes strategy to save lives. 2021. https://www.gov.uk/government/publications/ suicide-prevention-in-england-fifth-progress-report (accessed April 7, 2021).

19 Hawton K, Saunders K, Topiwala A, Haw C. Psychiatric disorders in patients presenting to hospital following self-harm: a systematic review. J Affect Disord 2013; 151: 821-30.

20 Gillies D, Christou MA, Dixon AC, et al. Prevalence and characteristics of self-harm in adolescents: meta-analyses of community-based studies 1990-2015. J Am Acad Child Adolesc Psychiatry 2018; 57: 733-41

21 Office for National Statistics. Ethnic group by sex by age. https:// www.nomisweb.co.uk/census/2011/dc2101ew (accessed Sept 8, 2020).

22 Ministry of Housing, Communities \& Local Government. The English Indices of Deprivation 2019. 2019. https://www.gov.uk/ government/statistics/english-indices-of-deprivation-2019 (accessed July 23, 2020).

23 Gunnell D, Bennewith O, Simkin S, et al. Time trends in coroners' use of different verdicts for possible suicides and their impact on officially reported incidence of suicide in England: 1990-2005. Psychol Med 2013; 43: 1415-22.

24 Carr MJ, Ashcroft DM, Kontopantelis E, et al. The epidemiology of self-harm in a UK-wide primary care patient cohort, 2001-2013. BMC Psychiatry 2016; 16: 53.

25 Patton GC, Coffey C, Romaniuk H, et al. The prognosis of common mental disorders in adolescents: a 14-year prospective cohort study. Lancet 2014; 383: 1404-11.

26 Bor W, Dean AJ, Najman J, Hayatbakhsh R. Are child and adolescent mental health problems increasing in the 21st century? A systematic review. Aust N Z J Psychiatry 2014; 48: 606-16.

27 NHS Digital. Mental health of children and young people in England, 2017. https://files.digital.nhs.uk/A0/273EE3/MHCYP\%20 2017\%20Trends\%20Characteristics.pdf (accessed Oct 14, 2020).

28 Fardouly J, Vartanian LR. Social media and body image concerns: current research and future directions. Curr Opin Psychol 2016; 9: 1-5.

29 Kelly Y, Zilanawala A, Booker C, Sacker A. Social media use and adolescent mental health: findings from the UK millennium cohort study. EClinicalMedicine 2019; 6: 59-68.

30 Hamm MP, Newton AS, Chisholm A, et al. Prevalence and effect of cyberbullying on children and young people: a scoping review of social media studies. JAMA Pediatr 2015; 169: 770-77.

31 Public Health England. Trends in hospital admissions for mental health conditions and as a result of self-harm in children and young people, by specific age groups and for local authorities. 2019. https://www.gov.uk/government/publications/mental-health-andself-harm-in-children-and-young-people (accessed Oct 22, 2020).

32 Hall S-M, McIntosh K, Neitzert E, et al. Intersecting inequalities: the impact of austerity on Black and minority ethnic women in the UK. 2017. https://wbg.org.uk/wp-content/uploads/2018/08/ Intersecting-Inequalities-October-2017-Full-Report.pdf (accessed July 23, 2020).

33 Kapur N, Steeg S, Webb R, et al. Does clinical management improve outcomes following self-harm? Results from the Multicentre Study of Self-Harm in England. PLoS oNE 2013; 8: e70434.

34 Vostanis P, Svirydzenka N, Dugard P, Singh S, Dogra N. Mental health service use by adolescents of Indian and White origin. Arch Dis Child 2013; 98: 764-67.

35 Vahdaninia M, Simkhada B, van Teijlingen E, Blunt H, Mercel-Sanca A. Mental health services designed for Black, Asian and Minority Ethnics (BAME) in the UK: a scoping review of case studies. MHSI 2020; 24: 81-95

36 Wasserman D, Hoven CW, Wasserman C, et al. School-based suicide prevention programmes: the SEYLE cluster-randomised, controlled trial. Lancet 2015; 385: 1536-44.

37 National Institute for Health and Care Excellence. Self-harm in over 8s: short-term management and prevention of recurrence. 2004. https://www.nice.org.uk/guidance/cg16 (accessed Feb 2, 2021). 